Network Working Group

Request for Comments: 3823

B. Kovitz

Category: Informational

MIME Media Type for the Systems Biology Markup Language (SBML)

Status of this Memo

This memo provides information for the Internet community. It does not specify an Internet standard of any kind. Distribution of this memo is unlimited.

Copyright Notice

Copyright (C) The Internet Society (2004).

Abstract

This document registers the MIME sub-type application/sbml+xml, a media type for SBML, the Systems Biology Markup Language. SBML is defined by The SBML Team at the California Institute of Technology and interested members of the systems biology community.

1. Introduction

SBML is an XML format for representing and exchanging models of biochemical reaction networks used in systems biology. SBML:

- enables researchers in systems biology to use multiple tools, such as simulators, editors, differential-equation solvers, and visualizers, on a single model without rewriting the model for each tool;

- enables researchers and publishers to make models available online to other researchers even if they use a different software environment;

o enables models, and the intellectual effort put into them, to survive beyond the lifetime of the software tools used to create them.

Currently, about 60 software applications use SBML, and researchers are using these applications to develop quantitative and qualitative computational models, mostly in cell biology. In addition, several consortia and alliances have standardized SBML as their model 
definition language. The SBML community hopes that a standardized MIME media type will help researchers share models on a larger scale, drawing more heavily on the capabilities of the world-wide web.

A detailed exposition of SBML and its uses within the systems biology community is available in references [HUCKA2003], [FINNEY2003], and [HUCKA2 004 ].

\section{IANA Registration}

This section registers application/sbml+xml as a MIME media type according to the parameters set forth in [RFC2048].

MIME media type name: application

MIME subtype name: sbml+xml

Required parameters: none.

Optional parameters: none.

There is no charset parameter. Character handling has identical semantics to the case where the charset parameter of the "application/xml" media type is omitted, as described in section 3.2 of [RFC3023]. Note that SBML level 2 is defined to have UTF-8 encoding [SBML2-1, section 4.1].

Encoding considerations:

Same as described in section 3.2 of [RFC3023].

Security considerations:

The security considerations described in section 7 of [RFC3470]

all potentially apply to sbml+xml documents. In particular, sbml+xml documents might contain the results of proprietary biological research that their owner may wish to keep private.

The XML schema for sbml+xml provides for no active or executable content.

Interoperability considerations:

The information in an sbml+xml document describes an abstract model of biochemical reactions. It is not tied to any particular software application, and indeed the primary purpose of SBML is to make these models readable and writable by many different software applications. 
This might seem to make sbml+xml more appropriate for the "Model" primary content type [RFC2077], but SBML models are not guaranteed to have the required three orthogonal dimensions. SBML models, rather, involve interacting entities that exist within compartments. However, ideally, browsers and other software that reads sbml+xml would give a human reader multiple choices of how to view the document: in a data-visualization tool, in a model editor, in a differential-equation analyzer, etc.

The systems biology community has and will continue to release new levels and versions of the SBML schema and semantics. New versions attempt to be backward compatible with old versions, but sometimes small incompatibilities are introduced. Every sbml+xml document contains its level and version; programs that read sbml+xml should read this information to be sure they correctly interpret the remainder of the document.

Published specification:

A list of all current SBML specifications and related documents is maintained at:

http://sbml.org/documents

Current specifications are:

SBML level 2, version 1

http://sbml.org/specifications/sbml-level-2-v1.ps

http://sbml.org/specifications/sbml-level-2-v1.pdf

SBML level 1, version 2

http://sbml.org/specifications/sbml-level-1-v2.ps

http://sbml.org/specifications/sbml-level-1-v2.pdf

SBML level 1, version 1

http://sbml.org/specifications/sbml-level-1-v1.ps

http://sbml.org/specifications/sbml-level-1-vl.pdf

All specifications are authored by and available in hardcopy form from The SBML Team (see below for mailing information).

Applications which use this media type:

The following application and database projects read and/or write models in sbml+xml format. Currently, most do not encode or decode MIME-format messages. Hopefully the registration of 


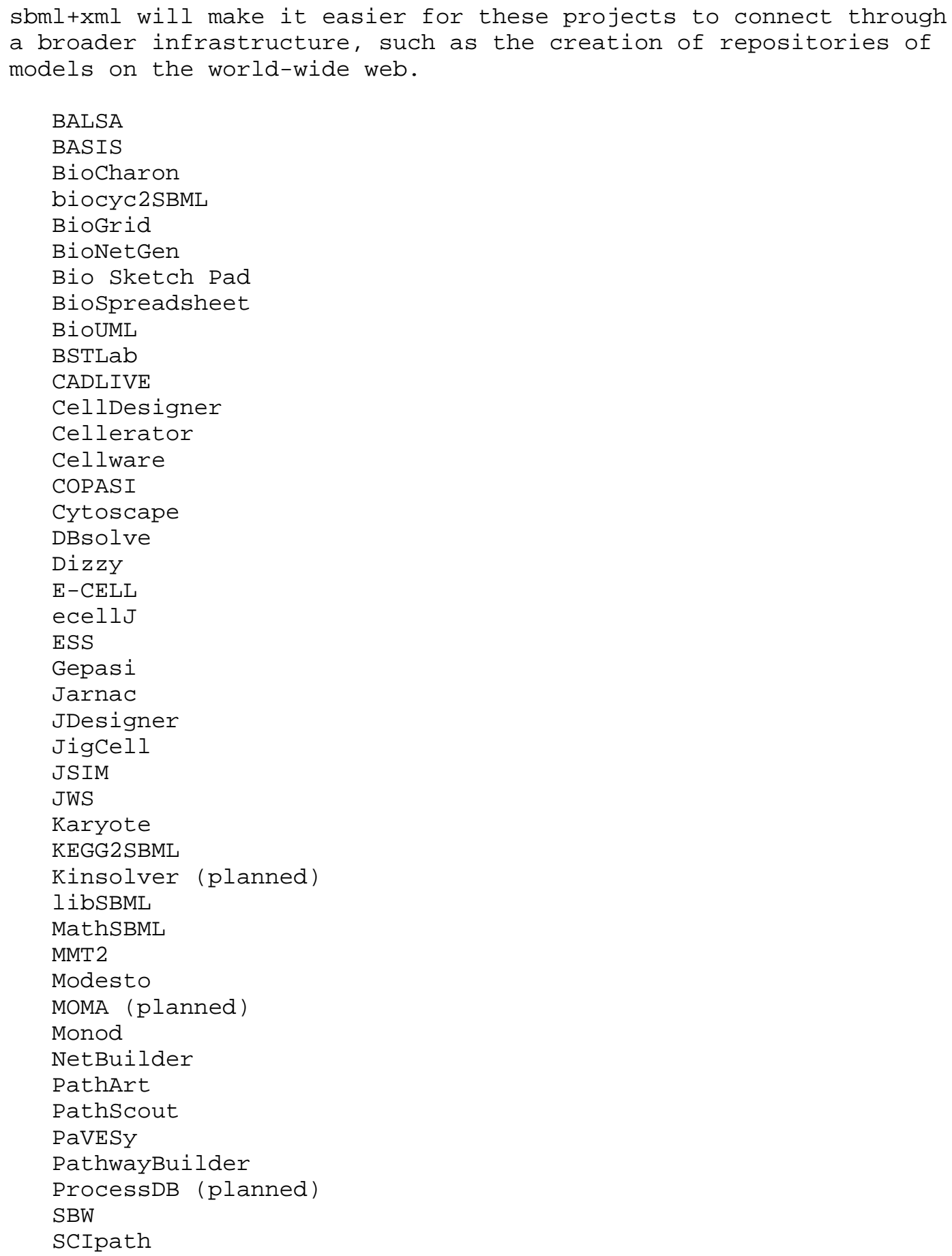




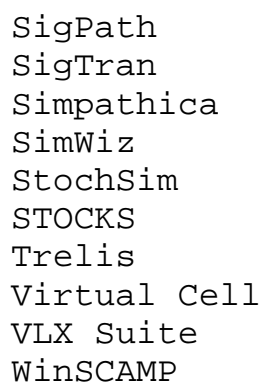

A list of SBML-enabled applications, along with URLs for more information about them, is maintained at http://sbml.org.

Additional information:

For further information, contact:

Michael Hucka

mhuckaecaltech.edu

Andrew Finney

afinneyecds.caltech.edu

The SBML Team

http://sbml.org

sbml-teamecaltech.edu

Control and Dynamical systems, MC 107-81

California Institute of Technology

Pasadena, CA 91125

USA

Intended usage: LIMITED USE

Author/Change Controller:

The SBML specification is a free, open, community effort organized and edited by The SBML Team. The SBML Team has change control over the specification.

The SBML Team and interested members of the systems biology community meet regularly at the "Workshops on Software Platforms for systems Biology". Information about past and planned workshops is maintained at:

http://sbml.org/workshops 


\section{Security Considerations}

Security considerations for sbml+xml are discussed in the "Security Considerations" heading in the IANA registration in section 2 .

4. Contributors

The following people contributed to the content of this document: Michael Hucka (Caltech), Andrew Finney (University of Hertfordshire).

5. References

\subsection{Normative References}

[SBML2-1] Finney, A. and Hucka, M., "Systems Biology Markup Language (SBML) Level 2: Structures and Facilities for Model Definitions", June 28, 2003. Available from The SBML Team at: http://sbml.org/specifications/sbmllevel-2-v1.pdf

[SBML1-2] Hucka, M., Finney, A., Sauro, H. and Bolouri, H., "Systems Biology Markup Language (SBML) Level 1: Structures and Facilities for Basic Model Definitions", August 28, 2003. Available from The SBML Team at: http://sbml.org/specifications/sbml-level-1-v2.pdf

\subsection{Informative References}

[FINNEY2003] Finney, A. and Hucka, M., "Systems Biology Markup Language: Level 2 and Beyond", Biochemical Society Transactions 31:1472-1473, December, 2003.

[HUCKA2003] Hucka, M., Finney, A., Sauro, H.M., Bolouri, H., Doyle, J.C., Kitano, H., Arkin, A.P., Bornstein, B.J., Bray, D., Cornish-Bowden, A., Cuellar, A.A., Dronov, S., Gilles, E.D., Ginkel, M., Gor, V., Goryanin, I.I., Hedley, W.J., Hodgman, T.C., Hofmeyr, J.-H., Hunter, P.J., Juty, N.S., Kasberger, J.L., Kremling, A., Kummer, U., Le Novere, N., Loew, L.M., Lucio, D., Mendes, P., Minch, E., Mjolsness, E.D., Nakayama, Y., Nelson, M.R., Nielsen, P.F., Sakurada, T., Schaff, J.C., Shapiro, B.E., Shimizu, T.S., Spence, H.D., Stelling, J., Takahashi, K., Tomita, M., Wagner, J., Wang, J., "The Systems Biology Markup Language (SBML): a Medium for Representation and Exchange of Biochemical Network Models", Bioinformatics 19(4):524-531, 2003. 
[HUCKA2004] Hucka, M., Finney, A., Bornstein, B. J., Keating, S. M., Shapiro, B. E., Matthews, M., Kovitz, B. L., Schilstra, M. J., Funahashi, A., Doyle, J. C., and Kitano, H., "Evolving a Lingua Franca and Associated Software Infrastructure for Computational Systems Biology: The Systems Biology Markup Language (SBML) Project", systems Biology, Vol. 1, 2004 .

[RFC2048] Freed, N., Klensin, J. and J. Postel, "Multipurpose Internet Mail Extensions (MIME) Part Four: Registration Procedures", BCP 13, RFC 2048, November 1996.

[RFC2077] Nelson, S. and C. Parks, "The Model Primary Content Type for Multipurpose Internet Mail Extensions", RFC 2077, January 1997 .

[RFC3023] Murata, M., St. Laurent, S. and D. Kohn, "XML Media Types", RFC 3023, January 2001.

[RFC3470] Hollenbeck, S., Rose, M. and L. Masinter, "Guidelines for the Use of Extensible Markup Language (XML) within IETF Protocols", BCP 70, RFC 3470, January 2003.

6. Author's Address

Ben Kovitz

Control and Dynamical Systems, MC 107-81

California Institute of Technology

Pasadena, CA 91125

USA

Phone: +1 626 395-6911

EMail: bkovitz@caltech.edu 
7. Full Copyright statement

Copyright (C) The Internet Society (2004). This document is subject to the rights, licenses and restrictions contained in BCP 78, and except as set forth therein, the authors retain all their rights.

This document and the information contained herein are provided on an "AS IS" basis and THE CONTRIBUTOR, THE ORGANIZATION HE/SHE REPRESENTS OR IS SPONSORED BY (IF ANY), THE INTERNET SOCIETY AND THE INTERNET ENGINEERING TASK FORCE DISCLAIM ALL WARRANTIES, EXPRESS OR IMPLIED, INCLUDING BUT NOT LIMITED TO ANY WARRANTY THAT THE USE OF THE INFORMATION HEREIN WILL NOT INERINGE ANY RIGHTS OR ANY IMPLIED WARRANTIES OF MERCHANTABILITY OR FITNESS FOR A PARTICULAR PURPOSE.

Intellectual Property

The IETF takes no position regarding the validity or scope of any Intellectual Property Rights or other rights that might be claimed to pertain to the implementation or use of the technology described in this document or the extent to which any license under such rights might or might not be available; nor does it represent that it has made any independent effort to identify any such rights. Information on the procedures with respect to rights in RFC documents can be found in BCP 78 and BCP 79 .

Copies of IPR disclosures made to the IETF Secretariat and any assurances of licenses to be made available, or the result of an attempt made to obtain a general license or permission for the use of such proprietary rights by implementers or users of this specification can be obtained from the IETF on-line IPR repository at http://www.ietf.org/ipr.

The IETF invites any interested party to bring to its attention any copyrights, patents or patent applications, or other proprietary rights that may cover technology that may be required to implement this standard. Please address the information to the IETF at ietfipraietf.org.

Acknowledgement

Funding for the RFC Editor function is currently provided by the Internet Society. 